\title{
Incidence of Infection among Subjects with Helicobacter pylori Seroconversion
}

\author{
Young Jung Kim ${ }^{1,2}$, Sun-Young Lee', Jeong Hwan Kim' ', In-Kyung Sung ${ }^{1}$, and Hyung Seok Park' \\ ${ }^{1}$ Department of Internal Medicine, Konkuk University School of Medicine, Seoul, ${ }^{2}$ Department of Internal Medicine, Chonnam University \\ School of Medicine, Gwangju, Korea
}

Background/Aims: Helicobacter pylori (H. pylori) seroconversion may occur during screening for gastric cancer. Our study aimed to assess the number of seroconverted subjects with $H$. pylori and their results in follow-up tests.

Methods: Data were consecutively collected on subjects who were $H$. pylori-seronegative and presented for gastric cancer screening. Subjects who were followed up using the same serology test and pepsinogen (PG) assays on the day of endoscopy were included in the study.

Results: During the follow-up of $57.7 \pm 21.4$ months, 61 (15.0\%) of 407 seronegative subjects showed seroconversion. H. pylori infection was detected in six (9.8\%) of 61 seroconverted subjects. A diffuse red fundal appearance, with a significant increase in the Kyoto classification scores for gastritis, was observed in the infected subjects $(p<0.001)$. Compared to the false-seropositive subjects, infected subjects showed higher serology titers $(p<0.001)$ and PG II levels $(p<0.001)$ and lower PG I/II ratios $(p=0.002)$, in the followup tests.

Conclusions: Seroconversion occurred in 3.3\% of seronegative subjects per year; however, only $9.8 \%$ had $H$. pylori infection. The majority $(90.2 \%)$ of the seroconverted subjects showed false seropositivity without significant changes in the follow-up test results. The diffuse red fundal appearance could be an indicator of $H$. pylori infection. Clin Endosc 2022;55:67-76

Key Words: Endoscopy; Gastritis; Helicobacter pylori; Screening

\section{INTRODUCTION}

Serum assays are often combined with gastroscopic examination in gastric cancer screening. Gastric biopsy allows for accurate detection of the presence of Helicobacter pylori; however, false-negative findings are not rare. ${ }^{1}$ Indeed, the risk of false-negative Giemsa staining results is increased in patients with decreased gastric secretory ability. ${ }^{2}$ Therefore, in addition to gastroscopy, serum anti-H. pylori immunoglobulin G (IgG)

Received: November 16, 2020 Revised: January 12, 2021

Accepted: March 3, 2021

Correspondence: Sun-Young Lee

Department of Internal Medicine, Konkuk University School of Medicine, 120-1

Neungdongro, Gwangjingu, Seoul 05030, Korea

Tel: +82-2-2030-7747, Fax: +82-2-2030-7748, E-mail: sunyoung@kuh.ac.kr

ORCID: https://orcid.org/0000-0003-4146-6686

(cc) This is an Open Access article distributed under the terms of the Creative Commons Attribution Non-Commercial License (http://creativecommons.org/ licenses/by-nc/3.0) which permits unrestricted non-commercial use, distribution, and reproduction in any medium, provided the original work is properly cited. titers are often monitored, along with serum pepsinogen (PG) levels. ${ }^{3}$ Although the specificity of the serology test was lower, the sensitivity was higher than in other $H$. pylori tests. ${ }^{4}$

Gastroscopy findings and serum assay results help predict the status of H. pylori infection. ${ }^{5}$ However, in an H. pylori-seroprevalent population, a considerable proportion (22.2\%) of seronegative subjects with normal PG assay findings had past infection. ${ }^{6}$ Moreover, seronegative subjects often demonstrate closed-type (22.8\%) or open-type (6.0\%) atrophic gastritis, even in the absence of gastric corpus atrophy (serum PG I $\leq 70 \mathrm{ng} / \mathrm{mL}$ and PGI/II $\leq 3){ }^{7}$ A diffuse red fundal appearance, mucosal swelling, nontransparent sticky mucus, and hypertrophic gastric rugae in the corpus are well-known findings of active $H$. pylori infection; alternatively, regular arrangement of collecting venules (RAC), linear streaks, and fundic gland polyps are more common in the absence of $H$. pylori infection. ${ }^{8}$

It is important to collect data of false-seropositive subjects without infection and those infected with $H$. pylori during the follow-up of seronegative individuals. A recent study indicated 
that the annual seroconversion rate was $2.79 \%$, and that living with an $\mathrm{H}$. pylori-infected individual was a risk factor for seroconversion in Koreans. ${ }^{9}$ It is important to accurately identify $H$. pylori infection among seroconverted subjects; however, data during screening for gastric cancer are limited in Korea. In this study, we aimed to accurately determine the number of subjects accurately diagnosed with $H$. pylori after seroconversion in follow-up tests. Furthermore, we aimed to identify the differences between $H$. pylori-infected subjects and those with false-seropositive results.

\section{SUBJECTS AND METHODS}

\section{Selection of study subjects}

After our previous study, we followed-up a consecutive series of Koreans subjects until 2019 who were H. pylori-seronegative and underwent screening for gastric cancer at our center between 2010 and 2014. ${ }^{10}$ Among the subjects registered at ClinicalTrials.gov (NCT01824953), only those who were followed up using the same $H$. pylori serologic test for $\geq 24$ months were analyzed in this study. Subjects with a recent history of $H$. pylori eradication within the past two years, renal failure, or a history of gastrectomy were excluded from the study.

This study was performed at our center in accordance with the Helsinki Declaration after Institutional Review Board approval (KUH1010626). Informed consent was obtained from the subjects before performing gastric cancer screening tests using GIF-H260, GIF-H290 (Olympus, Tokyo, Japan), or EG2990i (Pentax, Tokyo, Japan) endoscopes; serum PG assay (HBi Co., Anyang, Korea); and serum anti-H. pylori IgG Chorus assay (DIESSE Diagnostica Senese, Siena, Italy). All digital data were collected using ethical methods.

\section{Assessment for endoscopy and gastric biopsy findings}

Based on the Kyoto classification scoring system for gastritis, the presence and degree of chronic atrophic gastritis (A), metaplastic gastritis (IM), hypertrophic gastric rugae $(H)$, nodular gastritis $(\mathrm{N})$, and diffuse red (DR) fundal appearance were scored from 0 to $8 .{ }^{11}$ Briefly, chronic atrophic gastritis was scored as A0 (none or closed-type 1), A1 (closed-type 2 or 3), and A2 (open-type). Metaplastic gastritis was scored as IM0 (none), IM1 (limited), or IM2 (extensive). Hypertrophic rugae were scored as $\mathrm{H} 0$ (absent) and $\mathrm{H} 1$ (present). Nodular gastritis was scored as N0 (absent) or N1 (present). The diffuse red fundal appearance was scored as DR0 (none), DR1 (mild), and DR2 (severe).
Endoscopic biopsies were performed on lesions with abnormal shapes or discoloration. Gastric biopsy specimens were subjected to hematoxylin and eosin staining, as well as Giemsa staining. H. pylori-related gastric biopsy findings have been reported as previously described. ${ }^{12} \mathrm{H}$. pylori infection was confirmed based on positive Giemsa staining.

\section{Determining $H$. pylori infection status}

Infection status was classified as $H$. pylori-naïve, past, or current. Past infection was defined as either a history of eradication, $H$. pylori-related changes on gastroscopy, or gastric biopsy findings. In order to exclude subjects with spontaneous regression or unintended eradication, classification of $H$. pylori-naive stomachs was based on the following criteria: (1) no previous diagnosis of $H$. pylori infection, (2) negative findings on invasive and noninvasive $H$. pylori tests, (3) absence of gastric corpus atrophy (serum PG I $\leq 70 \mathrm{ng} / \mathrm{mL}$ and PG I/II ratio $\leq 3),(4)$ absence of gastric atrophy, intestinal metaplasia, neutrophil, or $\mathrm{H}$. pylori in the biopsied specimen (only a mild degree of mononuclear cell infiltration was permitted), and (5) regular arrangement of collecting venules on gastroscopy without evidence of past infection (either the presence of metaplastic gastritis, gastric xanthoma, or advanced atrophic gastritis $\geq$ closed-type 2$)^{13}$

\section{Interpretation of $\boldsymbol{H}$. pylori serologic test}

Seropositivity was diagnosed when anti-H. pylori IgG level was $\geq 12.0 \mathrm{AU} / \mathrm{mL}$, with $100 \%$ sensitivity and $75 \%$ specificity based on our previous study. ${ }^{14}$ Seroconversion was considered when a seronegative subject showed seropositive test findings in the follow-up tests. The subjects were finally classified into subjects without seroconversion (persistent seronegative group) and subjects with seroconversion (seropositive group).

\section{Primary outcome measurement}

Accurate detection of $\mathrm{H}$. pylori infection was considered the primary endpoint of this study. Current infection was diagnosed based on a positive Giemsa staining finding. Seronegative subjects with positive Giemsa staining findings were excluded, since non-H. pylori Helicobacter or Campylobacter may show false-positive Giemsa staining findings. ${ }^{15}$ In these cases, follow-up H. pylori tests (i.e., ${ }^{13} \mathrm{C}$-urea breath test [UBT] with the aid of the POCone ${ }^{\circledR}$ [Otsuka Electronics Co., Ltd., Osaka, Japan]) were performed for confirmation to verify new infection in $\mathrm{H}$. pylori-naïve subjects and to identify reinfection in subjects with past infection. Recrudescence was not considered in this study since all subjects with a history of successful eradication showed seronegative results for $\geq 24$ months after eradication of the remote $H$. pylori infection. 


\section{Secondary outcome measurement}

Seroconversion in follow-up tests was considered as the secondary endpoint. Seroconverted subjects without evidence of ongoing $H$. pylori infection were regarded as having false-positive test findings. The absence of infection after seroconversion was confirmed either by a negative ${ }^{13} \mathrm{C}$-UBT result or by spontaneous seroreversion of $H$. pylori with persistent RAC in follow-up tests.

\section{Statistical analysis}

Differences between the two groups were analyzed using the Student's $t$-test for continuous variables and the chi-square test for categorical variables. Continuous variables with symmetrical distribution are presented as mean \pm standard deviation, and categorical variables are presented as the number of subjects with proportion (\%). For continuous variables with asymmetrical distribution, data were presented as medians with ranges using the Kruskal-Wallis test. The Fisher's exact test was used for categorical variables with an asymmetrical distribution. Differences between the initial and follow-up tests were analyzed using the Wilcoxon signed-rank test. All statistical analyses were performed using PASW statistics (version 24.0; SPSS Inc., Chicago, IL, USA). Statistical significance was set at $p<0.05$.

\section{RESULTS}

\section{Characteristics of the included H. pylori- seronegative subjects}

A total of 407 seronegative subjects underwent follow-up tests using the same serum anti-H. pylori IgG Chorus assay. Baseline characteristics and test findings are summarized in Table 1.

\section{Incidence of seroconversion}

During the mean follow-up of $57.7 \pm 21.4$ months, 61 (15.0\%) of 407 seronegative subjects showed seroconversion (Fig. 1). The annual seroconversion rate was 3.3\%. The seroconverted subjects had a longer follow-up period $(68.6 \pm 23.8$ months $v s$. $55.8 \pm 20.4$ months, $p<0.001)$ and a higher initial serology titer than those without seroconversion $(6.7 \pm 1.6 \mathrm{AU} /$ $\mathrm{mL} v$ s. $5.7 \pm 1.3 \mathrm{AU} / \mathrm{mL}, p<0.001)$.

\section{Seropositive subjects with $H$. pylori infection in the follow-up tests}

H. pylori infection was detected in six (9.8\%) of 61 seroconverted subjects. Therefore, $H$. pylori infection was observed in $2.0 \%(0.42 \%$ per year) of seronegative subjects during screen- ing for gastric cancer. The initial and follow-up test results of the six infected subjects are summarized in Table 2.

Changes in endoscopic findings were observed in an $H$. pylori-naïve subject with new infection (Fig. 2), as well as in subjects with reinfection in whom eradication therapy was previously performed (Fig. 3), and in a subject with unintended eradication (Fig. 4). Compared to the initial endoscopic findings, all infected subjects demonstrated diffuse red fundal appearance. The total Kyoto classification scores $(p<0.001)$ and diffuse red fundal appearance scores $(p<0.001)$ increased significantly.

\section{False-seropositive subjects in the follow-up tests}

Compared to the initial serologic titers, follow-up serologic titers showed a significant increase from $6.4 \mathrm{AU} / \mathrm{mL}$ to 22.0 $\mathrm{AU} / \mathrm{mL}(p<0.001)$. However, there were no significant changes in the serum PG assay findings (Table 3). A small increase was observed in the Kyoto classification scores for gastritis (from 0.13 to $0.30, p=0.006$ ) and metaplastic gastritis scores (from 0.11 to $0.27, p=0.006$ ); however, none of these false-seropositive subjects exhibited a positive $H$. pylori test result in follow-up tests. ${ }^{13} \mathrm{C}$-UBT was performed in nine subjects, and it was negative in this group of subjects. Furthermore, 52 of 55 false-seropositive subjects showed persistent RAC on follow-up endoscopy. Spontaneous seroreversion of $H$. pylori was observed in 31 subjects who underwent the same serological follow-up procedure during screening for gastric cancer.

\section{Difference between the follow-up test findings in the infected and false-seropositive subjects}

The median serologic titer increased from $6.5 \mathrm{AU} / \mathrm{mL}$ to 132.7 AU/mL in the seropositive subjects with $H$. pylori infection $(p=0.021)$. Hence, the serology titer was significantly higher among the infected subjects than the false-seropositive subjects (132.7 AU/mL vs. $22.0 \mathrm{AU} / \mathrm{mL}, p<0.001$ ) at the time of seroconversion (Table 3). Moreover, serum PG II levels were higher in the infected group than in the false-seropositive group (22.8 $\pm 10.5 \mathrm{ng} / \mathrm{ml} v s .10 .4 \pm 5.3 \mathrm{ng} / \mathrm{ml}, p<0.001)$, and serum PG I/II ratios were lower in the infected group than in the false-seropositive group $(3.7 \pm 1.1 v s .6 .2 \pm 1.6, p=0.002)$.

In the follow-up endoscopies, the infected subjects showed a significant increase in the Kyoto classification scores (2.75 vs. $0.30, p<0.001)$, chronic atrophic gastritis scores $(0.60 v s .0 .04$, $p<0.001)$, metaplastic gastritis scores (1.00 vs. $0.27, p<0.001)$, and diffuse red fundal appearance scores $(1.33 v s .0, p<0.001)$ (Table 3). All infected subjects had diffuse red fundal appearance; meanwhile, this finding was not observed in any of the false-seropositive subjects $(p<0.001)$. 
Table 1. Baseline Characteristics and Test Findings of the 407 Helicobacter pylori-Seronegative Subjects

\begin{tabular}{|c|c|c|c|c|}
\hline \multirow[b]{2}{*}{ Variables } & \multirow{2}{*}{$\begin{array}{l}\text { All subjects } \\
(n=407)\end{array}$} & \multicolumn{3}{|c|}{ Follow-up serology test findings } \\
\hline & & $\begin{array}{l}\text { Without seroconversion } \\
\qquad(n=346)\end{array}$ & $\begin{array}{l}\text { With seroconversion } \\
\qquad(n=61)\end{array}$ & $p$-value \\
\hline Age (years) & $50.9 \pm 10.0$ & $50.7 \pm 10.0$ & $52.2 \pm 9.8$ & 0.279 \\
\hline Sex (male) & $253(62.1 \%)$ & $211(61.0 \%)$ & $42(68.9 \%)$ & 0.243 \\
\hline Body mass index $\left(\mathrm{kg} / \mathrm{m}^{2}\right)$ & $24.3 \pm 3.1$ & $24.3 \pm 3.2$ & $24.8 \pm 2.9$ & 0.255 \\
\hline Past H. pylori infection & $155(38.1 \%)$ & $127(36.7 \%)$ & $28(45.9 \%)$ & 0.173 \\
\hline H. pylori eradication history & $127(31.2 \%)$ & $102(29.5 \%)$ & $25(41.0 \%)$ & 0.074 \\
\hline Unintended eradication $^{\mathrm{a}}$ & $28(6.9 \%)$ & $25(7.2 \%)$ & $3(4.9 \%)$ & 0.783 \\
\hline \multicolumn{5}{|l|}{ Comorbidities } \\
\hline Diabetes mellitus & $50(12.3 \%)$ & $41(11.8 \%)$ & $9(14.8 \%)$ & 0.542 \\
\hline Hypertension & $113(27.8 \%)$ & $93(26.9 \%)$ & $20(32.8 \%)$ & 0.342 \\
\hline Coronary heart disease & $25(6.1 \%)$ & $18(5.2 \%)$ & $7(11.5 \%)$ & 0.078 \\
\hline \multicolumn{5}{|l|}{ Recent drug intake } \\
\hline Nonsteroidal anti-inflammatory drug & $32(7.9 \%)$ & $26(7.5 \%)$ & $6(9.8 \%)$ & 0.604 \\
\hline Antithrombotic agents including aspirin & $49(12.0 \%)$ & $42(12.1 \%)$ & $7(11.5 \%)$ & 0.883 \\
\hline Acid suppressants & $5(1.2 \%)$ & $4(1.2 \%)$ & $1(1.6 \%)$ & 0.558 \\
\hline \multicolumn{5}{|l|}{ Initial test findings } \\
\hline Serum anti-H. pylori IgG (AU/mL) & $5.9 \pm 1.4$ & $5.7 \pm 1.3$ & $6.7 \pm 1.6$ & $<0.001$ \\
\hline Serum pepsinogen I (ng/ml) & $52.6 \pm 21.7$ & $51.8 \pm 20.1$ & $56.9 \pm 28.8$ & 0.092 \\
\hline Serum pepsinogen II (ng/ml) & $9.8 \pm 4.3$ & $9.5 \pm 4.0$ & $10.4 \pm 5.4$ & 0.139 \\
\hline Serum pepsinogen I/II ratio & $5.6 \pm 1.4$ & $5.6 \pm 1.4$ & $5.7 \pm 1.5$ & 0.934 \\
\hline Total follow-up period (months) & $57.7 \pm 21.4$ & $55.8 \pm 20.4$ & $68.6 \pm 23.8$ & $<0.001$ \\
\hline \multicolumn{5}{|l|}{ Follow-up test findings } \\
\hline Serum anti-H. pylori IgG (AU/mL) & $6.1(5-200)$ & $5.6(5-11.9)$ & $22.7(12.1-200)$ & $<0.001$ \\
\hline Serum pepsinogen I (ng/ml) & $55.7 \pm 25.1$ & $54.5 \pm 23.1$ & $62.5 \pm 31.5$ & 0.022 \\
\hline Serum pepsinogen II (ng/ml) & $8.4(3-36.6)$ & $8.3(3-36.6)$ & $9.6(3.8-35.6)$ & 0.001 \\
\hline Serum pepsinogen I/II ratio & $6.3 \pm 2.0$ & $6.4 \pm 2.1$ & $6.0 \pm 1.7$ & 0.197 \\
\hline
\end{tabular}

H. pylori, Helicobacter pylori; IgG, immunoglobulin G.

Continuous variables are presented as mean \pm standard deviation using the $t$-test, and categorical variables are presented as number of the subjects with proportion (\%) using the Chi-square test. For continuous variables with asymmetrical distribution, data are presented as median with ranges using the Kruskal-wallis test. For categorical variables with asymmetrical distribution, Fisher's exact test was used.

${ }^{a}$ Unintended eradication or spontaneous regression of $H$. pylori was considered when intestinal metaplasia, advanced atrophic gastritis, or gastric xanthoma was observed in subjects without eradication history. 


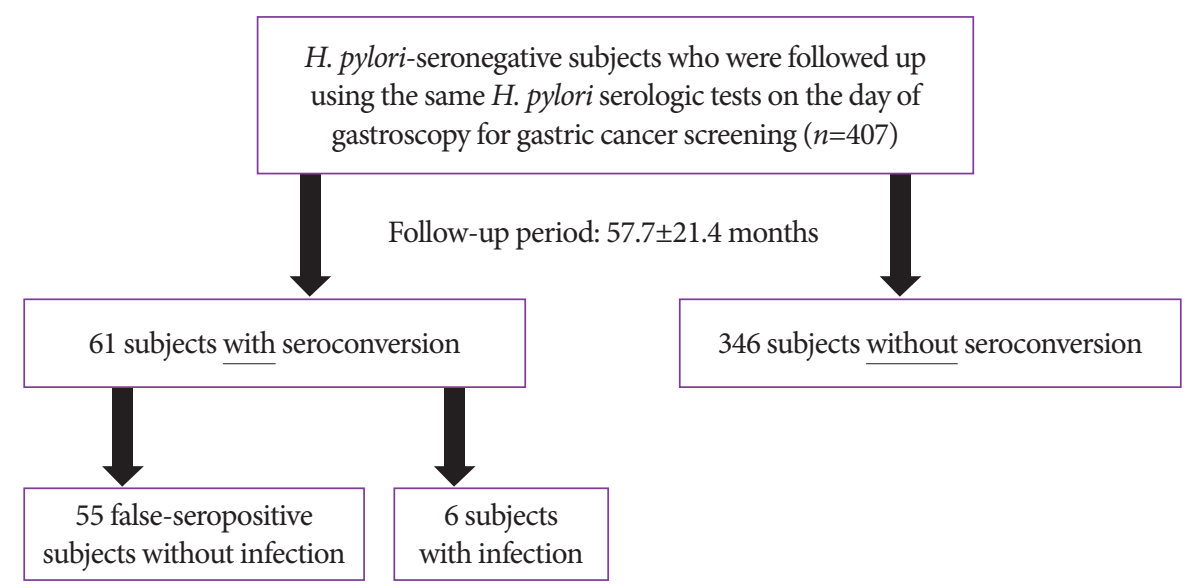

Fig. 1. Flowchart of this study. A total of 407 consecutive Helicobacter pylori (H. pylori)-seronegative subjects were included in our study. During the mean follow-up period of $57.7 \pm 21.4$ months, 61 subjects showed seroconversion; meanwhile, seronegative results persisted in 346 subjects. H. pylori infection was observed in six $(9.8 \%)$ of 61 seroconverted subjects.

Table 2. Initial and Follow-Up Test Findings of the Six Seroconverted Subjects with Helicobacter pylori Infection

\begin{tabular}{|c|c|c|c|c|c|c|c|c|c|c|c|c|}
\hline \multirow{2}{*}{$\begin{array}{l}\text { Initial infection } \\
\text { status }\end{array}$} & \multirow{2}{*}{$\begin{array}{l}\text { Sex } / \\
\text { age }\end{array}$} & \multirow{2}{*}{$\begin{array}{l}\text { Comor- } \\
\text { bidity }\end{array}$} & \multicolumn{2}{|c|}{$\begin{array}{l}\text { Serology } \\
(\mathrm{AU} / \mathrm{mL})\end{array}$} & \multicolumn{2}{|c|}{$\begin{array}{c}\text { PG I } \\
(\mathbf{n g} / \mathrm{ml})\end{array}$} & \multicolumn{2}{|c|}{$\begin{array}{c}\text { PG II } \\
(\mathrm{ng} / \mathrm{ml})\end{array}$} & \multicolumn{2}{|c|}{ PG I/II ratio } & \multicolumn{2}{|c|}{$\begin{array}{c}\text { Kyoto classification score } \\
\text { for gastritis }^{\mathrm{a}}\end{array}$} \\
\hline & & & Initial & $F / U$ & Initial & $\mathbf{F} / \mathbf{U}$ & Initial & $F / U$ & Initial & $\mathrm{F} / \mathrm{U}$ & Initial & $\mathrm{F} / \mathrm{U}$ \\
\hline H. pylori-naïve & $\mathrm{M} / 55$ & DM, HTN & 6.4 & 21.7 & 69.5 & 85.1 & 18.9 & 22.5 & 3.7 & 3.8 & $\begin{array}{c}0 \text { (A0 IM0 } \\
\text { H0 N0 } \\
\text { DR0) }\end{array}$ & $\begin{array}{c}2 \text { (A0 IM0 } \\
\text { H0 N0 } \\
\text { DR2) }\end{array}$ \\
\hline \multirow[t]{4}{*}{$\begin{array}{l}\text { Previous eradica- } \\
\text { tion history }\end{array}$} & $\mathrm{M} / 54$ & HTN & 9.4 & 161.5 & 46.4 & 97.2 & 10.3 & 29.8 & 4.5 & 3.3 & $\begin{array}{c}3 \text { (A1 IM2 } \\
\text { H0 N0 } \\
\text { DR0) }\end{array}$ & $\begin{array}{c}5 \text { (A1 IM2 } \\
\text { H0 N0 } \\
\text { DR2) }\end{array}$ \\
\hline & $\mathrm{F} / 67$ & $\begin{array}{l}\text { HTN, os- } \\
\text { teoporosis }\end{array}$ & 6.5 & $>200$ & 45.2 & 116.0 & 10.5 & 35.6 & 4.3 & 3.3 & $\begin{array}{c}1 \text { (A0 IM1 } \\
\text { H0 N0 } \\
\text { DR0) }\end{array}$ & $\begin{array}{c}2 \text { (A0 IM1 } \\
\text { H0 N0 } \\
\text { DR1) }\end{array}$ \\
\hline & $\mathrm{F} / 66$ & $\begin{array}{l}\text { DM, } \\
\text { HTN, } \\
\text { CVA }\end{array}$ & 9.3 & 19.2 & 39.2 & 42.7 & 8.1 & 7.6 & 4.9 & 5.6 & $\begin{array}{c}2 \text { (A1 IM1 } \\
\text { H0 N0 } \\
\text { DR0) }\end{array}$ & $\begin{array}{c}3 \text { (A1 IM1 } \\
\text { H0 N0 } \\
\text { DR1) }\end{array}$ \\
\hline & $\mathrm{M} / 49$ & None & 5.8 & 107.2 & 33.7 & 66.1 & 6.3 & 27.8 & 5.3 & 2.4 & $\begin{array}{c}1 \text { (A0 IM1 } \\
\text { H0 N0 } \\
\text { DR0) }\end{array}$ & $\begin{array}{c}4 \text { (A2 IM1 } \\
\text { H0 N0 } \\
\text { DR1) }\end{array}$ \\
\hline $\begin{array}{l}\text { Unintended } \\
\text { eradication }^{\mathrm{b}}\end{array}$ & $\mathrm{M} / 53$ & None & $<5$ & 158.1 & 58.5 & 54.3 & 12.2 & 13.6 & 4.8 & 4.0 & $\begin{array}{c}1 \text { (A0 IM1 } \\
\text { H0 N0 } \\
\text { DR0) }\end{array}$ & $\begin{array}{c}2 \text { (A0 IM1 } \\
\text { H0 N0 } \\
\text { DR1) }\end{array}$ \\
\hline
\end{tabular}

CVA, cerebrovascular disease; DM, diabetes mellitus; F, female; F/U, follow-up; H. pylori, Helicobacter pylori; HTN, hypertension; M, male; PG, pepsinogen.

${ }^{a}$ Chronic atrophic gastritis was scored as A0 (none or closed-type 1), A1 (closed-type 2 or 3), and A2 (open-type). Metaplastic gastritis was scored as IM0 (none), IM1 (limited), and IM2 (extensive). Hypertrophic rugae were scored as H0 (absent) and H1 (present). Nodular gastritis was scored as N0 (absent) and N1 (present). Diffuse redness was scored as DR0 (none), DR1 (mild), and DR2 (severe).

${ }^{\mathrm{b}}$ Unintended eradication was considered in this subject because intestinal metaplasia was observed in the gastric biopsied specimen. 

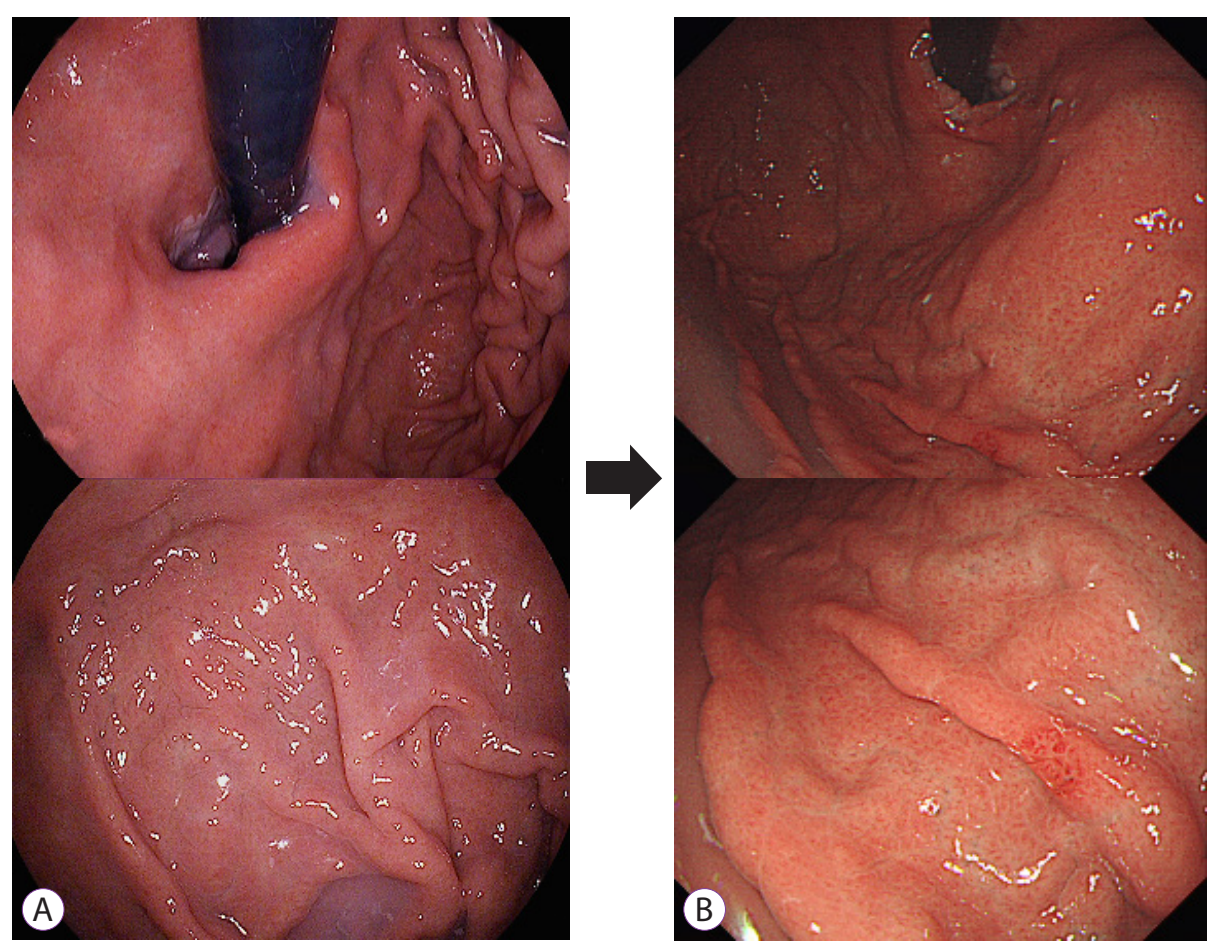

Fig. 2. Development of diffuse red fundal appearance on follow-up endoscopy in an Helicobacter pylori (H. pylori)-naïve subject with new infection. (A) Initial endoscopic findings of the fundus and cardia. The Kyoto classification score was 0 (A0, IMO, HO, N0 DRO). Serum pepsinogen (PG) and serology assays showed normal results, as summarized in Table 2. (B) Findings of follow-up endoscopy performed after four years. Multiple tiny hemorrhagic spots were observed in the fundus with positive Giemsa staining. The Kyoto classification score increased from 0 to 2 (A0 IMO HO N0 DR2). Seroconversion in H. pylori antibodies (6.4 $\rightarrow 21.7 \mathrm{AU} / \mathrm{mL}$ ) was reported with higher serum PG I $(69.5 \rightarrow 85.1 \mathrm{ng} / \mathrm{ml})$ and PG II $(18.9 \rightarrow 22.5 \mathrm{ng} / \mathrm{ml})$ levels compared to the initial test findings.
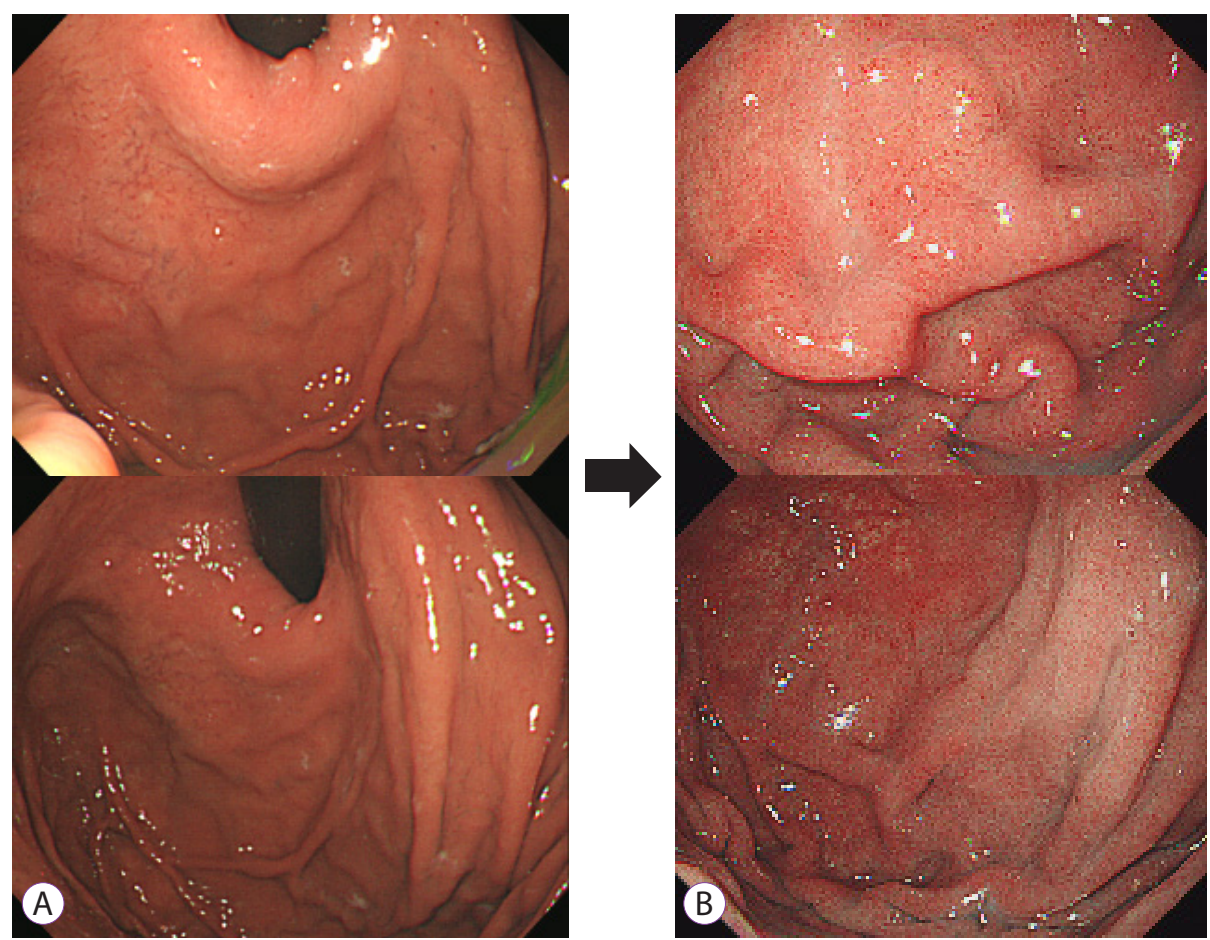

Fig. 3. Changes in the endoscopic findings of a subject who had a history of successful eradication four years prior to the initial visit. (A) Endoscopic findings of the fundus at the initial visit. The Kyoto classification score was 3 (A1 IM2 H0 N0 DRO). Successful eradication was confirmed by negative ${ }^{13} \mathrm{C}$-urea breath test findings. (B) Findings of follow-up endoscopy performed after six years. The development of diffuse red fundal appearance and positive Giemsa staining were observed. The Kyoto classification score increased from 3 to 5 (A1 IM2 H0 N0 DR2) with seroconversion $(9.4 \rightarrow 161.5 \mathrm{AU} / \mathrm{mL}$ ). The serum pepsinogen (PG) I (46.4 $\rightarrow 97.2 \mathrm{ng} / \mathrm{ml})$ and PG II $(10.3 \rightarrow 29.8 \mathrm{ng} / \mathrm{ml})$ levels were higher compared to the initial test results, whereas the PG I/I ratio was lower $(4.5 \rightarrow 3.3)$. 

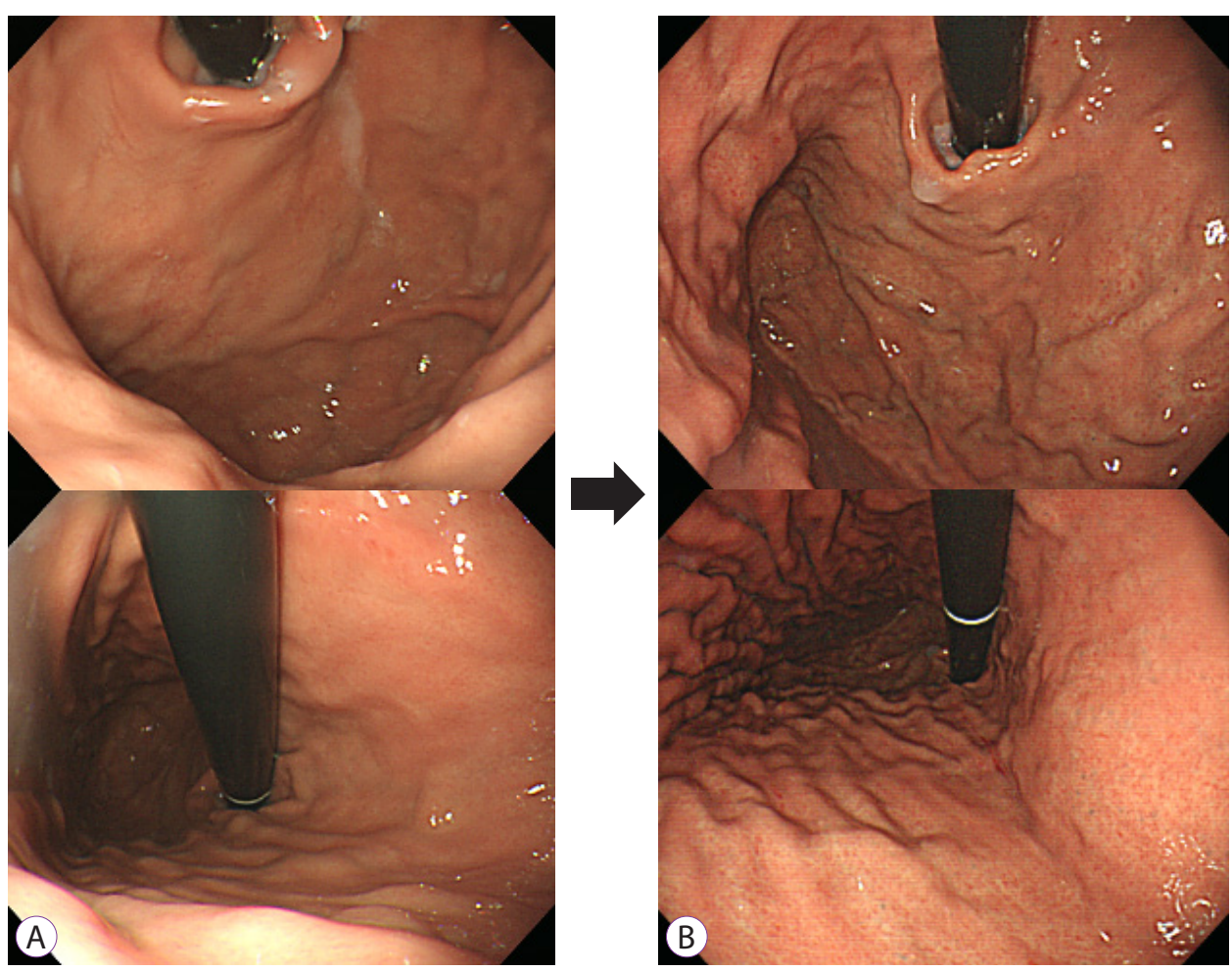

Fig. 4. Findings of initial and follow-up endoscopy in a subject with unintended eradication prior to the initial visit. (A) Initial endoscopic findings of the fundus. Unintended eradication of Helicobacter pylori was considered in this study since intestinal metaplasia was observed in the biopsied specimens obtained from the antrum. Serum pepsinogen and serology assays showed normal findings, and the Kyoto classification score was 1 (A0, IM1, H0, N0, DR0). (B) Follow-up endoscopy performed after four years revealed diffuse red fundal appearance and enlarged gastric pit openings in the fundus. Positive Giemsa staining results were obtained with seroconversion ( $<5 \rightarrow$ 158.1). The Kyoto gastritis classification score increased to two (A0 IM1 H0 N0 DR1).

Table 3. Difference between the Findings of Initial and Follow-Up Tests in the 61 Seroconverted Subjects

\begin{tabular}{|c|c|c|c|c|c|c|c|}
\hline \multirow[t]{2}{*}{ Findings } & \multicolumn{3}{|c|}{$\begin{array}{l}\text { False-seropositive subjects without } \\
\text { infection }(n=55)\end{array}$} & \multicolumn{3}{|c|}{$\begin{array}{l}\text { Seropositive subjects with } H \text {. pylori } \\
\text { infection }(n=6)\end{array}$} & \multirow[t]{2}{*}{$p$-value ${ }^{\mathrm{b}}$} \\
\hline & Initial & Follow-up & $p$-value ${ }^{\mathrm{a}}$ & Initial & Follow-up & $p$-value ${ }^{a}$ & \\
\hline Serum anti-H. pylori $\operatorname{IgG}(\mathrm{AU} / \mathrm{mL})$ & $6.4(5-9.9)$ & $22.0(12.1-59.3)$ & $<0.001$ & $6.5(5-9.4)$ & $132.7(19.2-200)$ & 0.021 & $<0.001$ \\
\hline Serum pepsinogen I (ng/ml) & $57.8 \pm 30.0$ & $60.9 \pm 31.9$ & 0.383 & $48.8 \pm 13.1$ & $76.9 \pm 27.6$ & 0.062 & 0.547 \\
\hline Serum pepsinogen II (ng/ml) & $10.3 \pm 5.5$ & $10.4 \pm 5.3$ & 0.991 & $11.1 \pm 4.4$ & $22.8 \pm 10.5$ & 0.054 & $<0.001$ \\
\hline Serum pepsinogen I/II ratio & $5.8 \pm 1.5$ & $6.2 \pm 1.6$ & 0.076 & $4.6 \pm 0.6$ & $3.7 \pm 1.1$ & 0.153 & 0.002 \\
\hline Total Kyoto classification score & $0.13(0-2)$ & $0.30(0-2)$ & 0.006 & $1.25(0-3)$ & $2.75(2-5)$ & 0.004 & $<0.001$ \\
\hline Chronic atrophic gastritis score & $0.04(0-2)$ & $0.04(0-2)$ & NA & $0.33(0-1)$ & $0.60(0-2)$ & 0.363 & 0.001 \\
\hline Metaplastic gastritis score & $0.11(0-2)$ & $0.27(0-2)$ & 0.006 & $1.00(0-2)$ & $1.00(0-2)$ & NA & 0.017 \\
\hline Diffuse redness score & 0 & 0 & NA & 0 & $1.33(1-2)$ & 0.001 & $<0.001$ \\
\hline Interval till seropositivity (months) & - & $36.8 \pm 16.1$ & - & - & $47.8 \pm 17.9$ & - & 0.302 \\
\hline
\end{tabular}

H. pylori, Helicobacter pylori; IgG, immunoglobulin G; NA, not available due to the absence of changes between the initial and follow-up test findings.

Continuous variables are presented as mean \pm standard deviation using the $t$-test, and those with asymmetrical distribution are presented as median with ranges using the Kruskal-wallis test.

${ }^{a} P$-values on the differences between the initial and follow-up test findings using the Wilcoxon signed-rank test.

${ }^{\mathrm{b}} P$-values on the differences in follow-up test findings between the false-seropositive and infected subjects. 


\section{DISCUSSION}

In this study, we found that only $9.8 \%$ of the seroconverted subjects were accurately diagnosed with $H$. pylori infection. Although seroconversion occurred annually in $3.3 \%$ of the seronegative subjects during screening for gastric cancer, the majority (90.2\%) of the subjects showed false-positive results without infection. A new $H$. pylori infection was observed in only $2.0 \%$ of the seronegative subjects during the mean follow-up period of 58 months ( $0.42 \%$ per year). All infected subjects showed diffuse redness in the fundus; however, it was not observed in any of the false-seropositive subjects. Therefore, diffuse redness in follow-up endoscopies could be considered an indicator for accurate detection of infection.

The seroconversion rate was related to a higher initial $H$. pylori titer and a longer follow-up period. Our findings are in accordance with those of a recent study that reported a link between high negative titers and the risk of gastric cancer based on the degree of atrophic gastritis. ${ }^{16}$ Moreover, follow-up serologic titers were useful for identifying infected subjects in this study. The serologic titers were significantly higher in the infected subjects than in the false-seropositive subjects. These findings are consistent with a recent study that demonstrated the usefulness of serum anti- $H$. pylori IgG titers for detection of reinfection. ${ }^{17}$ In summary, a high-negative IgG titer warrants serologic screening due to the higher risk of seroconversion and gastric cancer. Meanwhile, an increase in serologic titers in follow-up tests is indicative of ongoing $\mathrm{H}$. pylori infection.

In this study, it was beneficial to include the results of the serum PG assay, as well as those of the serological tests. The serum assay was useful in discriminating subjects with $H$. pylori-infection from false-seropositive subjects. Increased serum PG II levels and serum anti-H. pylori IgG titers, as well as decreased PG I/II ratios, were observed in the infected subjects in follow-up tests. Previous studies have shown that increased PG II levels are more specific for detecting active $H$. pylori infection than PG I levels, ${ }^{18}$ and PG I levels increase frequently due to drugs, including aspirin. ${ }^{19}$ Differences in serum PG values between the eradicated and non-infected subjects were not detected at $\geq 24$ months after successful eradication. ${ }^{20}$ High serum PG I and PG II levels indicate hyperchlorhydric stomachs, ${ }^{21}$ whereas low serum PG I levels and PG I/II ratios indicate hypochlorhydric stomachs with extensive atrophy. ${ }^{22}$ Consistent with these studies, PG II levels were significantly higher in infected subjects than in false-seropositive subjects; hence, the PG I/II ratio was significantly lower in infected subjects in this study. Serum assays cannot replace endoscopy for gastric cancer detection in a seroprevalent population; howev- er, they are useful in discriminating between subjects at highrisk for diffuse-type gastric cancer (i.e., high-seropositive IgG titers with high PG II levels) and those with intestinal-type gastric cancer or gastric adenoma (i.e., low PG I levels with low PG I/II ratios), neuroendocrine tumors (i.e., extremely low PG I levels in patients with autoimmune gastritis, or high PG I levels in patients with Zollinger-Ellison syndrome), and $H$. pylori-negative gastric cancers. ${ }^{23}$

Another notable finding of our study was diffuse red fundal appearance in infected subjects during follow-up endoscopy. Diffuse red fundal appearance represents the presence of infection, which progressively decreases after successful eradication. ${ }^{24}$ Increased hemoglobin index of the fundic mucosa during active inflammation appears to induce the diffuse red fundal appearance, since active inflammation due to $H$. pylori occurs more aggressively in the corpus than in the antrum. ${ }^{25} \mathrm{~A}$ recent study using ${ }^{13} \mathrm{C}$-UBT showed that the endoscopic Kyoto classification of gastritis was useful for diagnosing $H$. pylori infection, even in seronegative subjects with high serology titers. ${ }^{26}$ Furthermore, the Kyoto classification scoring system was useful in predicting reinfection in subjects in which the infection had been previously eradicated. ${ }^{27}$ The mean Kyoto score was 4.63 in subjects with active $H$. pylori infection; ${ }^{28}$ meanwhile, it was close to 0 in non-infected subjects in previous studies. ${ }^{29}$ The latter study showed that the endoscopy-based Kyoto score could detect false-seropositive subjects without infection, since the Kyoto score was 0 in $63.2 \%$ of the seropositive subjects with low seropositive titers. Consistent with these studies, infected subjects in our study showed a significant increase in the Kyoto classification scores at follow-up endoscopies. The development of diffuse red fundal appearance and positive Giemsa staining were detected in infected subjects, whereas none of these findings were observed in subjects with false-seropositive results.

There are some limitations in our study. For example, $H$. $p y$ lori infection was diagnosed based on positive Giemsa staining in seropositive subjects, and ${ }^{13} \mathrm{C}$-UBT was performed only in subjects who required confirmation. Most false-seropositive results were confirmed by spontaneous seroreversion and persistent RAC observed in follow-up endoscopies. Also, although image-enhanced endoscopy is useful for discriminating between $H$. pylori-active and inactive gastritis, it was not routinely performed in all screening endoscopies. ${ }^{30}$ Despite these limitations, we observed a diffuse red fundal appearance with higher Kyoto classification scores, H. pylori serology titers, and PG II levels in the infected subjects compared to the false-seropositive subjects.

In conclusion, seroconversion occurred in $3.3 \%$ of the seronegative subjects annually; however, only $9.8 \%$ were accurately 
diagnosed with $H$. pylori infection. The majority (90.2\%) of the seroconverted subjects showed false positive results with lower serology titers, lower serum PG II levels, and higher PG I/II ratios compared to the infected subjects. Moreover, spontaneous seroreversion and RAC were observed in false-seropositive subjects; meanwhile, a significant increase in the Kyoto classification scores for gastritis was observed in the infected subjects due to a diffuse red fundal appearance. Active $H$. pylori infection should be considered in the presence of diffuse red fundal appearance during screening for gastric cancer.

Conflicts of Interest

The authors have no potential conflicts of interest.

Funding

This study was supported by the National Research Foundation (NRF) of Korea, funded by the Ministry of Education (NRF2016R1D1A1B02008937).

\section{Author Contributions}

Conceptualization: Sun-Young Lee

Data curation: Young Jung Kim, SYL, Jeong Hwan Kim, In-Kyung Sung, and Hyung Seok Park

Formal analysis: YJK, SYL

Funding acquisition: SYL

Investigation: YJK, SYL, JHK, IKS, HSP

Methodology: TJK, SYL, JHK, IKS, HSP

Project administration: SYL

Resources: YJK, SYL, JHK, IKS, HSP

Software: SYL

Supervision: SYL

Validation: YJK, SYL, JHK, IKS, HSP

Visualization: YJK, SYL, JHK, IKH, HSP

Writing-original draft: $\mathrm{YJK}$

Writing-review\&editing: SYL

\section{ORCID}

Young Jung Kim

Sun-Young Lee

Jeong Hwan Kim

In-Kyung Sung

Hyung Seok Park

\begin{abstract}
https://orcid.org/0000-0003-4571-6021 https://orcid.org/0000-0003-4146-6686 https://orcid.org/0000-0002-2503-2688 https://orcid.org/0000-0002-3848-5571 https://orcid.org/0000-0003-3141-4858
\end{abstract}

\section{REFERENCES}

1. Shin CM, Kim N, Lee HS, et al. Validation of diagnostic tests for Helicobacter pylori with regard to grade of atrophic gastritis and/or intestinal metaplasia. Helicobacter 2009;14:512-519.

2. Kim JH, Lee SY, Lee SP, et al. The histologic detection of Helicobacter pylori in seropositive subjects is affected by pathology and secretory ability of the stomach. Helicobacter 2018;23:e12480.

3. Tu H, Sun L, Dong X, et al. Serum anti-Helicobacter pylori immunoglobulin $\mathrm{G}$ titer correlates with grade of histological gastritis, mucosal bacterial density, and levels of serum biomarkers. Scand J Gastroenterol 2014;49:259-266.

4. Bosch DE, Krumm N, Wener MH, et al. Serology is more sensitive than urea breath test or stool antigen for the initial diagnosis of Helicobacter pylori gastritis when compared with histopathology. Am J Clin Pathol 2020;154:255-265.

5. Lee SP, Lee SY, Kim JH, Sung IK, Park HS, Shim CS. Link between serum pepsinogen concentrations and upper gastrointestinal endoscopic findings. J Korean Med Sci 2017;32:796-802.

6. Kwon H, Lee SY, Kim JH, et al. ABC classification is less useful for older Koreans born before 1960. Gut Liver 2019;13:522-530.

7. Kishino T, Oyama T, Tomori A, Takahashi A, Shinohara T. Usefulness and limitations of a serum screening system to predict the risk of gastric cancer. Intern Med 2020;59:1473-1480.

8. Yoshii S, Mabe K, Watano K, et al. Validity of endoscopic features for the diagnosis of Helicobacter pylori infection status based on the Kyoto classification of gastritis. Dig Endosc 2020;32:74-83.

9. Jung JH, Choi KD, Han S, et al. Seroconversion rates of Helicobacter pylori infection in Korean adults. Helicobacter 2013;18:299-308.

10. Choi HS, Lee SY, Kim JH, et al. Combining the serum pepsinogen level and Helicobacter pylori antibody test for predicting the histology of gastric neoplasm. J Dig Dis 2014;15:293-298.

11. Kim GH. Endoscopic findings of Kyoto classification of gastritis. Korean J Helicobacter Up Gastrointest Res 2019;19:88-93.

12. Han HS, Lee SY, Oh SY, Moon HW, Cho H, Kim JH. Correlations of the gastric and duodenal microbiota with histological, endoscopic, and symptomatic gastritis. J Clin Med 2019;8:312.

13. Sugimoto M, Ban H, Ichikawa H, et al. Efficacy of the Kyoto classification of gastritis in identifying subjects at high risk for gastric cancer. Intern Med 2017;56:579-586.

14. Lee SY, Moon HW, Hur M, Yun YM. Validation of western Helicobacter pylori IgG antibody assays in Korean adults. J Med Microbiol 2015;64:513-518.

15. Seo TH, Lee SY, Uchida T, et al. The origin of non-Helicobacter pylori-related positive Giemsa stain in human gastric biopsy specimens. Dig Liver Dis 2011;43:23-27.

16. Inoue M, Sawada N, Goto A, et al.; JPHC Study Group. High-negative anti-Helicobacter pylori IgG antibody titers and long-term risk of gastric cancer: Results from a large-scale population-based cohort study in Japan. Cancer Epidemiol Biomarkers Prev 2020;29:420-426.

17. Lee JH, Kim N, Chung JI, et al. Long-term follow up of Helicobacter pylori IgG serology after eradication and reinfection rate of $\mathrm{H}$. pylori in South Korea. Helicobacter 2008;13:288-294.

18. Massarrat S, Haj-Sheykholeslami A, Mohamadkhani A, et al. Pepsinogen II can be a potential surrogate marker of morphological changes in corpus before and after $\mathrm{H}$. pylori eradication. Biomed Res Int 2014;2014:481607.

19. Shan J, Lei H, Shi W, Sun X, Tang Y, Ren C. High serum pepsinogen I and beta Helicobacter pylori infection are risk factors for aspirin-induced gastroduodenal injury. Dig Dis 2018;36:66-71.

20. Noh G, Kim N, Choi Y, et al. Long-term follow up of serum pepsinogens in subjects with gastric cancer or dysplasia after Helicobacter pylori eradication. J Gastroenterol Hepatol 2020;35:1540-1548.

21. Iijima K, Koike T, Ara N, et al. Identification of a high-risk group for low-dose aspirin-induced gastropathy by measuring serum pepsinogen in H. pylori-infected subjects. J Gastroenterol 2015;50:305-312.

22. Song HJ, Jang SJ, Yun SC, et al. Low levels of pepsinogen I and pepsinogen $\mathrm{I} / \mathrm{II}$ ratio are valuable serologic markers for predicting extensive gastric corpus atrophy in patients undergoing endoscopic mucosectomy. Gut Liver 2010;4:475-480.

23. Lee SY. Helicobacter pylori-negative gastric cancer. Korean J Helicobacter Up Gastrointest Res 2020;21:10-21.

24. Kato M, Terao S, Adachi K, et al.; Study Group for Establishing Endoscopic Diagnosis of Chronic Gastritis. Changes in endoscopic findings of gastritis after cure of $\mathrm{H}$. pylori infection: multicenter prospective trial. Dig Endosc 2013;25:264-273.

25. Kato T, Yagi N, Kamada T, Shimbo T, Watanabe H, Ida K; Study Group 
for Establishing Endoscopic Diagnosis of Chronic Gastritis. Diagnosis of Helicobacter pylori infection in gastric mucosa by endoscopic features: a multicenter prospective study. Dig Endosc 2013;25:508-518.

26. Toyoshima O, Nishizawa T, Arita M, et al. Helicobacter pylori infection in subjects negative for high titer serum antibody. World J Gastroenterol 2018;24:1419-1428.

27. Otani K, Watanabe T, Kosaka S, et al. Utility of Kyoto classification of gastritis in subjects with a high-negative titer of anti-Helicobacter pylori antibody during a medical check-up. J Clin Biochem Nutr 2020;67:317322.
28. Toyoshima O, Nishizawa T, Yoshida S, et al. Endoscopy-based Kyoto classification score of gastritis related to pathological topography of neutrophil activity. World J Gastroenterol 2020;26:5146-5155.

29. Nishizawa T, Sakitani K, Suzuki H, et al. A combination of serum anti-Helicobacter pylori antibody titer and Kyoto classification score could provide a more accurate diagnosis of H. pylori. United European Gastroenterol J 2019;7:343-348.

30. Osawa H, Miura Y, Takezawa T, et al. Linked color imaging and blue laser imaging for upper gastrointestinal screening. Clin Endosc 2018;51:513-526. 